\title{
Die altpaläolithischen Artefaktfunde von Uichteritz, Ldkr. Weißenfels
}

\author{
Armin Rudolph, Thomas Laurat \& Wolfgang Bernhardt *)
}

Keywords: Lower Palaeolithic flint artefacts, Middle Pleistocene gravel, Saale region, Saxony-Anhalt

Kurzfassung: In diesem Beitrag wird eine kleine Kollektion von Feuersteinartefakten aus mittelpleistozänen Schottern der Saale vorgestellt. Die Funde wurden in den Jahren seit 1994 in einer Kiesgrube bei Uichteritz geborgen. Geologischen Untersuchungen zufolge gehören die artefaktführenden Schotter wahrscheinlich in die späte Elster-Kaltzeit. Damit handelt es sich bei den Funden möglicherweise um die ältesten im mitteldeutschen Raum. Darüber hinaus gehören die von Uichteritz sowie von einer benachbarten Fundstelle bei Markröhlitz vorliegenden altpaläolithischen Artefakte noch immer zu den seltenen Funden aus dieser Zeit in Mitteldeutschland.

\section{[Lower Palaeolithic artefact finds from Uichteritz, near Weißenfels]}

Abstract: This report deals with a small collection of flint artefacts found in Middle Pleistocene gravels of the river Saale. The finds were recovered in a gravel pit near the village of Uichteritz between 1994 and 2004. According to geological analysis, the artefact bearing gravel complex was probably deposited in the Late Elsterian cold stage. Thus, the artefacts possibly belong to the oldest finds in Central Germany. In addition to this, the Lower Palaeolithic finds of Uichteritz and the neighbouring site of Markröhlitz

*Anschrift der Verfasser: A. Rudolph, Landsberger Str. 60 a, 04157 Leipzig. T. LaUrat, Landesamt für Denkmalpflege und Archäologie Sachsen-Anhalt, Richard-Wagner-Straße 9-10, 06114 Halle/Saale. W. Bernhardt, Hallesche Str. 59, 04435 Schkeuditz. are part of a find category which is still rare from that time in this area.

\section{Lage der Fundstelle}

Die Fundstelle, die Kiesgrube von H. Antons \& Sohn ${ }^{1}$, liegt etwa $4 \mathrm{~km}$ westlich von Weißenfels (Sachsen-Anhalt) auf dem Hang unmittelbar südlich des Röhlitzbaches, nordwestlich der Ortsmitte von Uichteritz. Ca. 1,2 km vom heutigen Saalelauf entfernt, werden in der relativ kleinen Kiesgrube mittelpleistozäne Schotter der Saale aufgeschlossen und abgebaut. Es handelt sich um die bereits lange bekannten Ablagerungen des Markröhlitzer Tales, das hier annähernd in west-östlicher Richtung verlief (WEISSERMEL 1908, Siegert \& Weissermel 1911, Schulz 1962, 1963). Der Aufschluß befindet sich in einem südlichen Bereich, aber nicht unmittelbar am südlichen Talrand des ehemals vielleicht ca. $2 \mathrm{~km}$ breiten mittelpleistozänen Saaletales.

\section{Fundgeschichte}

Bei einer ersten archäologischen Begehung in der Kiesgrube konnte W. Bernhardt im Rahmen seiner Tätigkeit als ehrenamtlicher Beauftragter des Landesamtes für Archäologie Sachsen-Anhalt, Halle (Saale), am 3.11.1994 auf der Überkornhalde vier Feuersteinabschläge finden. 

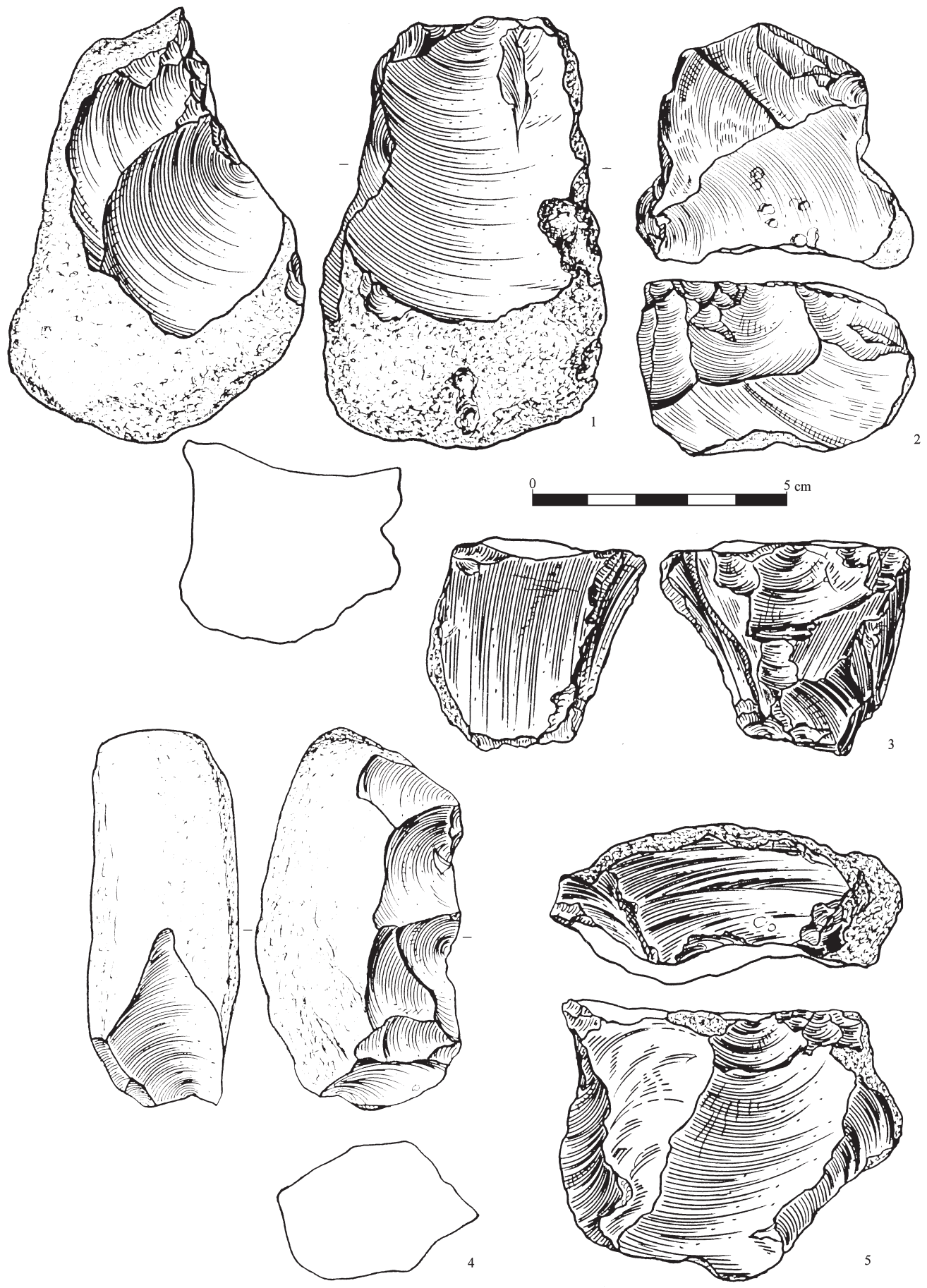

Abb. 1: Uichteritz, Ldkr. Weißenfels. Feuersteinartefakte: Kerne (1-3, 5), Chopper (4).

Fig. 1: Uichteritz, Ldkr. Weißenfels. Flint artefacts: cores (1-3, 5), chopper (4). 
In den Jahren bis 2004 wurden etwa 20 weitere Begehungen durchgeführt, die der Suche nach Artefakten und zum Teil auch der Klärung geologischer Fragen dienten ${ }^{2}$. Bisher konnte jedoch nur ein kleiner Komplex von lithischen Funden geborgen werden, was wohl in erster Linie mit ihrem seltenen Vorkommen in den abgebauten Schottern, z.T. aber auch mit dem geringen Umfang des Kiesabbaus im Aufschluß zusammenhängen wird. Die meisten Artefakte fanden sich auf Überkornhalden. Drei Abschläge und ein gebuchtetes Stück wurden in abgerutschten Kiesen an den steilen Böschungen entdeckt. Das gebuchtete Stück (Abb. 3, 4) und einer oder zwei der Abschläge lagen in Kiesen, die aus dem unteren Teil der feuersteinführenden Schotter, dem Mittleren Schotter nach Meng \& WANSA (2005; in diesem Heft) stammen. Ein weiterer Abschlag (Abb. 2, 3) konnte direkt im Verwitterungsbereich des Mittleren Schotters aufgefunden werden. Zumindest ein Teil der vorliegenden Feuersteinartefakte kann also dem nach Meng \& Wansa (2005) wahrscheinlich spätelsterkaltzeitlichen Mittleren Schotter zugewiesen werden. $\mathrm{Ob}$ ein weiterer Teil vielleicht auch aus dem Oberen Schotter stammt, läßt sich vorerst nicht sagen. An der Zeitstellung des Fundmaterials würde sich nichts Wesentliches ändern, da wahrscheinlich auch dieser Schotter in der späten Elster-Kaltzeit abgelagert wurde (Meng \& Wansa 2005).

Neben den Steinartefakten wurden auch mehrere Fragmente von Großsäugerknochen geborgen: ein unbestimmbares Diaphysen-Fragment wohl eines langen Röhrenknochens, ein kleines unbestimmbares Knochenfragment sowie 2 weitere unbestimmbare Knochenfragmente, die - nach der Stärke der Kompakta zu urteilen - wohl von einem Tier in der Größe eines Elefanten stammen ${ }^{3}$. Als Neufund liegt ein Backenzahn vom Pferd vor. Außerdem konnten in verschiedenen Bereichen der aufgeschlossenen Schotter Mollusken entdeckt werden. Eine systematische Entnahme von Molluskenproben erfolgte dann ab 2001 im Rahmen der detaillierten geologischen Untersuchungen durch $S$. Meng und S. Wansa, beide Halle (Saale), deren Ergebnisse im vorstehenden Aufsatz publiziert sind.

\section{Die Steinartefakte}

Bisher konnten 39 Steinartefakte gefunden wer$\mathrm{den}^{4}$. Sie wurden ausschließlich aus nordischem Feuerstein gefertigt und sind zumeist mehr oder weniger stark abgerollt. Nur geringfügig abgerollte und relativ scharfkantige Stücke kommen seltener vor. Die Artefakte besitzen überwiegend eine glänzende bräunliche bis gelbbräunliche oder gelbliche Patina. Außerdem wurden mehrere hellblau-weißlich patinierte Stücke gefunden.

Abschläge sind am häufigsten vertreten (Abb. 2, 2-6). 20 Exemplare liegen vor, darunter zwei beschädigte Stücke und ein Abschlaggerät. Die Längen der Abschläge schwanken erheblich und reichen von 18 bis $85 \mathrm{~mm}$, wobei über 40 $\mathrm{mm}$ lange Stücke am häufigsten vorkommen. Auch die Form ist sehr variabel. So kommen triangulär divergierende bzw. konvergierende, ovale und parallelseitige Abschläge vor. Die Symmetrieachse weicht dabei häufig von der Schlagrichtung ab, so daß asymmetrische Formen entstehen. Einmal liegt eine recht grobe Breitklinge vor (Abb. 2, 5). Die Bulben sind in der Regel sehr markant ausgeprägt. Der Schlagflächenrest ist häufig flach und trägt meist natürliche Spaltflächen. Singulär ist ein Abschlag mit einem bearbeiteten Schlagflächenrest. Da dieser aber nur eine Negativfläche trägt, ist unklar, ob er von einem vorher präparierten Kern stammt. Sehr deutlich sind grobe Schlagaugen ausgeprägt, die Durchmesser von 3 bis $7 \mathrm{~mm}$ haben. Teilweise sind bis zu 4 Augen - und ebenso viele Bulben - vorhanden, die anzeigen, dass der erste ausgeführte Schlag nicht immer zum Erfolg 

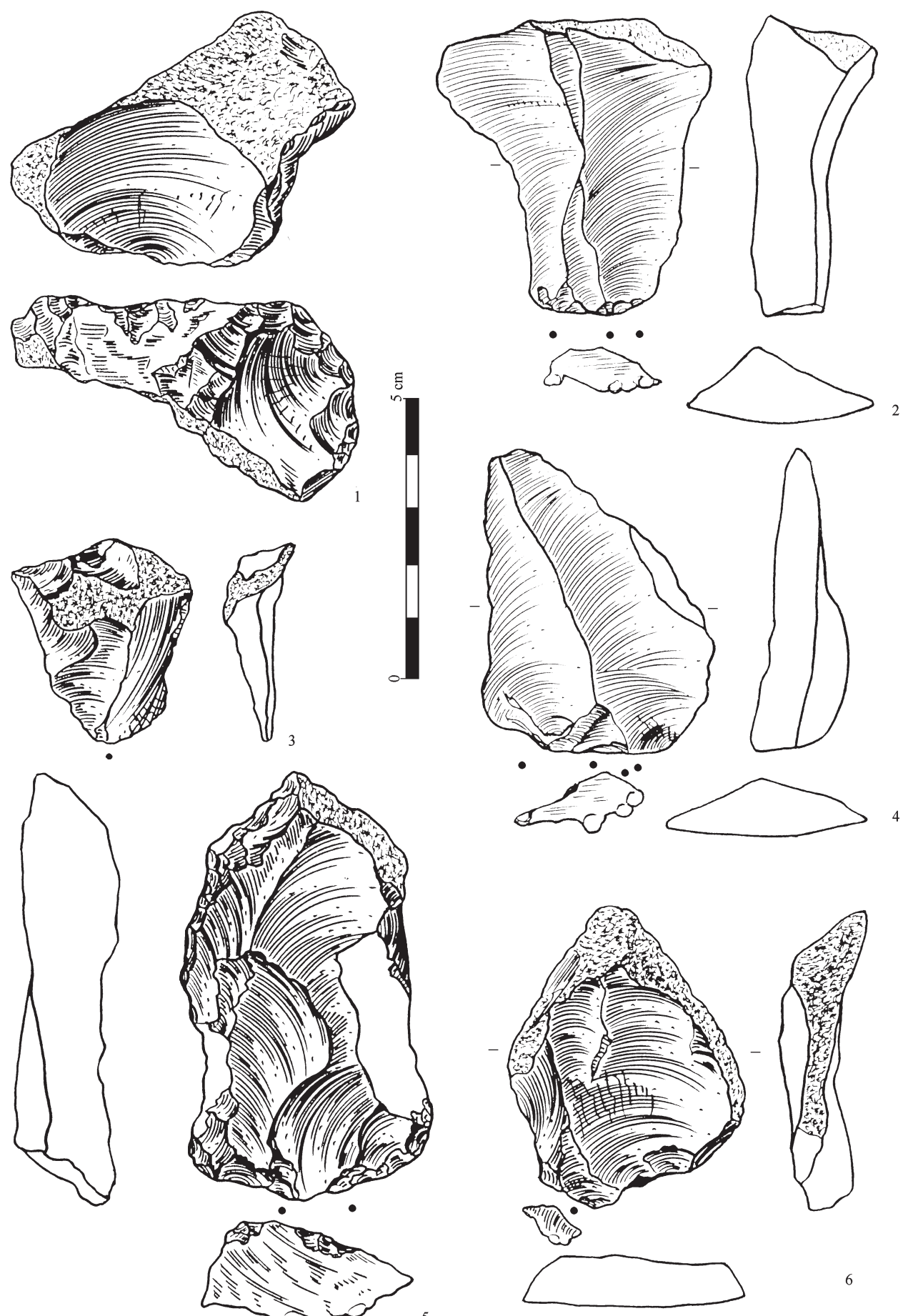

Abb. 2: Uichteritz, Ldkr. Weißenfels. Feuersteinartefakte: Kern (1), Abschläge (2-6).

Fig. 2: Uichteritz, Ldkr. Weißenfels. Flint artefacts: core (1), flakes (2-6). 

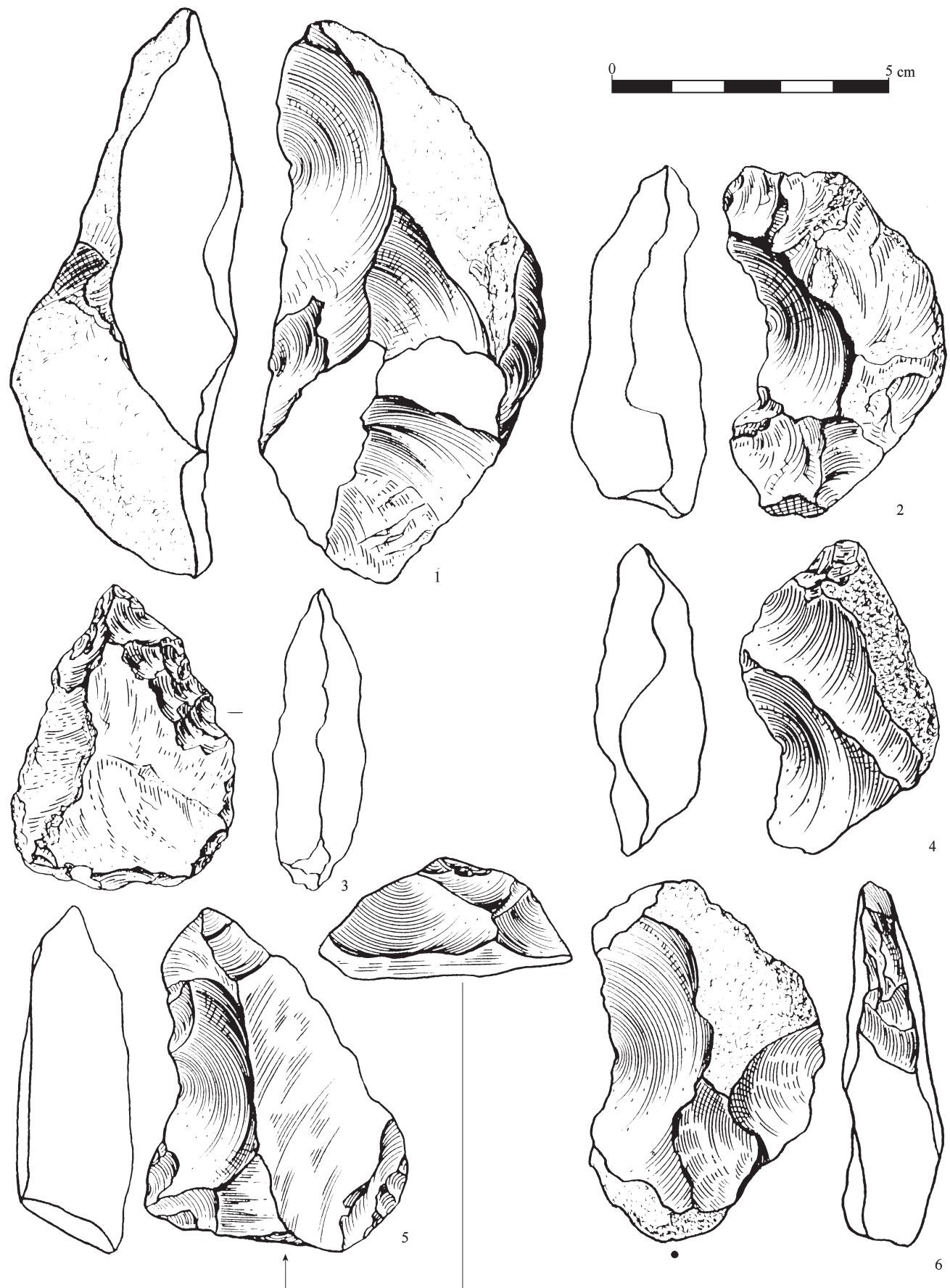

Abb. 3: Uichteritz, Ldkr. Weißenfels. Feuersteingeräte: gebuchtete Stücke (1-2, 4-6), retuschiertes Stück (3).

Fig. 3: Uichteritz, Ldkr. Weißenfels. Flint tools: notched pieces (1-2, 4-6) and retouched piece (3). 
führte. Der Winkel zwischen Schlagflächenrest und Ventralseite ist mit 110 bis $125^{\circ}$ recht groß. Aufmerksamkeit verdient auch ein Abschlag, der in der Quinson-Schlagtechnik hergestellt wurde. Abschläge ähnlichen Typs können in den umfangreicheren altpaläolithischen Inventaren von Bilzingsleben (Mania 1997) und Wallendorf (Laurat, Rudolph \& Bernhardt 2004) häufiger beobachtet werden. Dorsal sind häufig die Negativbahnen der vorhergehenden Abschläge zu sehen. Dabei kommen bis zu 7 Negative vor, die wechselnde Schlagrichtungen anzeigen. Fast die Hälfte aller Abschläge trägt dorsal Reste der Cortex. Echte Cortex- oder Primärabschläge wurden allerdings bisher sehr selten gefunden.

Kerne sind bisher durch 11 Exemplare vertreten (Abb. 1, 1-3. 5 und 2, 1). Sie erreichen Längen von 39 bis $136 \mathrm{~mm}$. Es überwiegen solche mit einer Abbau- und Schlagfläche, viermal liegen Stücke mit zwei Abbau- und Schlagflächen vor. Vorhergehende Präparationen beider Flächen sind nicht zu beobachten. Dreimal wurde die Schlagfläche durch einen Abschlag künstlich geschaffen. In einigen Fällen wurden vorher abgebaute Flächen als Schlagfläche genutzt (Abb. 1, 1. 2). Die Form der Kerne ist nicht festgelegt. Es kommen prismatische, strunkförmige und globulöse Kerne vor. Als Ausgangsstücke dienten offensichtlich natürliche Flussgerölle oder Stücke, die in angeschnittener Grundmoräne gefunden wurden. Kein Kern ist vollständig abgebaut, was daran zu erkennen ist, daß häufig noch erhebliche Cortexreste auf der Abbaufläche zu finden sind.

Die neun vorliegenden Geräte wurden überwiegend aus Trümmerstücken gefertigt, nur einmal wurde ein Abschlag modifiziert. Die Modifikationen sind grob und beschränken sich auf Clacton-Buchten (Abb. 3, 1-2, 4-6), denen gelegentlich eine kurze Retusche nachfolgen kann (Abb. 3, 2). Ein Stück mit mehreren aneinander gereihten groben Clacton-Buchten kann als Chopper angesprochen werden (Abb.
1, 4). Außerdem liegt eine partiell bogenförmig retuschierte Frostscherbe vor (Abb. 3, 3). Die Geräte besitzen eine Länge von 41 bis $101 \mathrm{~mm}$. Die Ansprache als Gerät ist nicht in allen Fällen zweifelsfrei, einige können auch Kerne repräsentieren. Der hohe Abrollungsgrad der Artefakte lässt eine Untersuchung auf Gebrauchsspuren nicht zu.

Die nicht sehr umfangreiche Artefaktkollektion lässt nur wenige Aussagen über Technologie und Typologie der Steinbearbeitung zu. Die wahrscheinlich spätelsterkaltzeitliche Altersstellung des Schotters weist bereits auf ein altpaläolithisches Inventar hin. Das Fehlen von Präparationstechniken, die meist groben, clactonoiden Abschläge und die einfachen Modifikationen scheinen diese Einordnung zu bestätigen. In seinem Gesamtcharakter ähnelt das Material jenem des Fundgebietes von Wallendorf (Laurat, Rudolph \& Bernhardt 2004), welches knapp $20 \mathrm{~km}$ in nordöstlicher Richtung entfernt liegt.

\section{Weitere altpaläolithische Funde aus dem Markröhlitzer Tal}

Beim Studium geologischer Karten und Literatur (Weissermel 1908; Siegert \& Weissermel 1911; Schulz 1962, 1963) war J. Thum, seinerzeit Hänichen, auf die östlich von Markröhlitz an beiden Talhängen des Röhlitzbaches ausstreichenden mittelpleistozänen Schotter der Saale aufmerksam geworden. Bei einer ersten Begehung am 8.5.1985 konnte er $1 \mathrm{~km}$ östlich von Markröhlitz am nördlichen Talrand die ersten Artefakte auf der Feldfläche entdecken. In den Jahren bis 1988 erfolgten weitere Begehungen durch ihn und z.T. durch T. Weber. Insgesamt konnten dabei knapp 30 Feuersteinartefakte als Oberflächenfunde oder aus einem Rohrgraben bzw. im Aushubmaterial dieses Grabens geborgen werden. Neben 

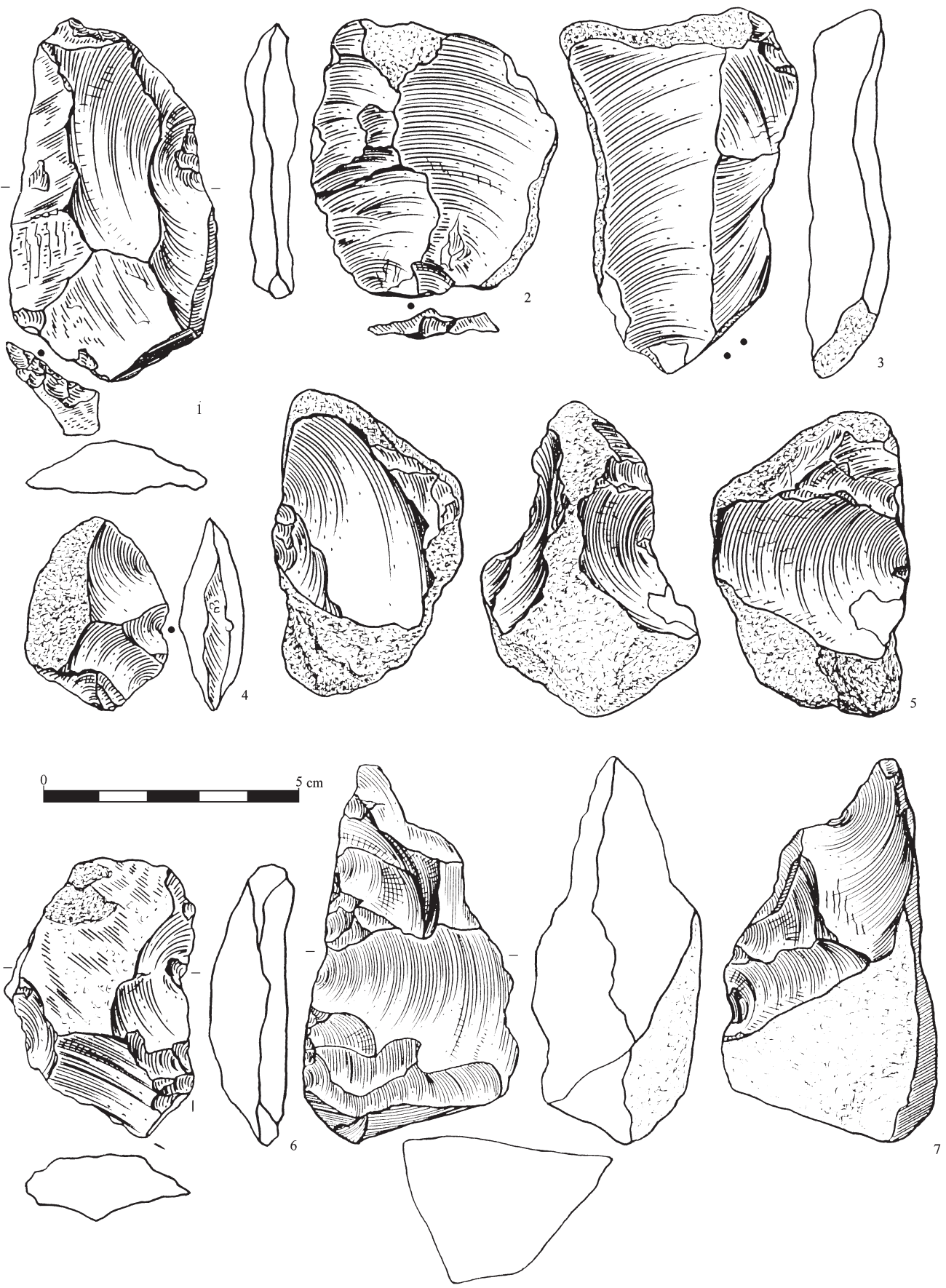

Abb. 4: Markröhlitz, Ldkr. Weißenfels. Feuersteinartefakte: Abschläge (1-4), Kern (5), retuschiertes Stück (6), Trieder (7).

Fig. 4: Markröhlitz, Ldkr. Weißenfels. Flint artefacts: flakes (1-4), core (5), retouched flake (6), trihedral (7). 

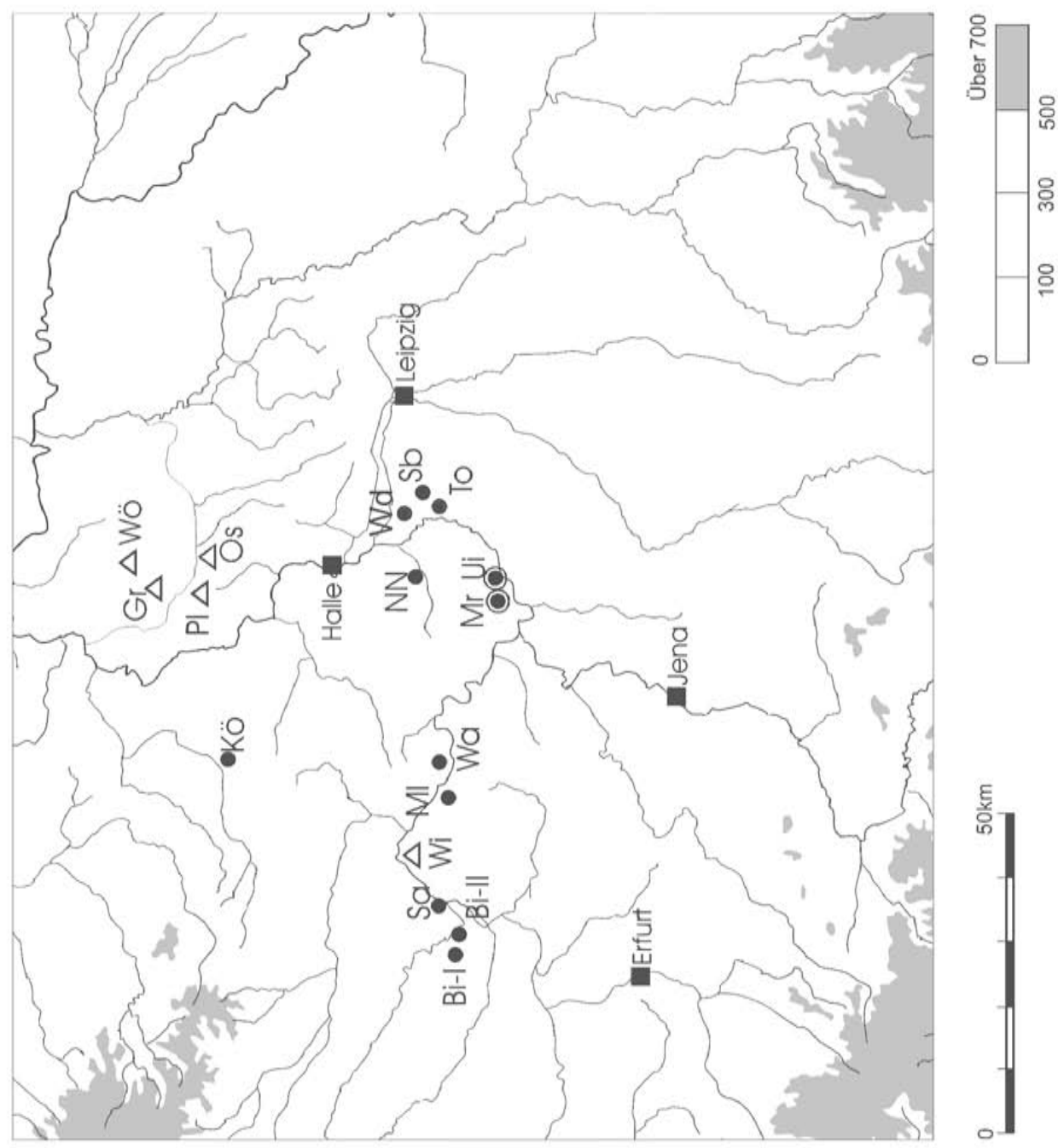

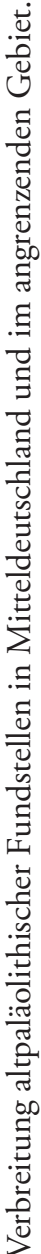

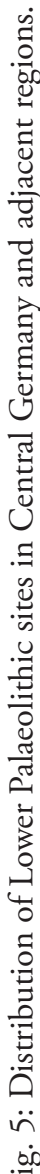

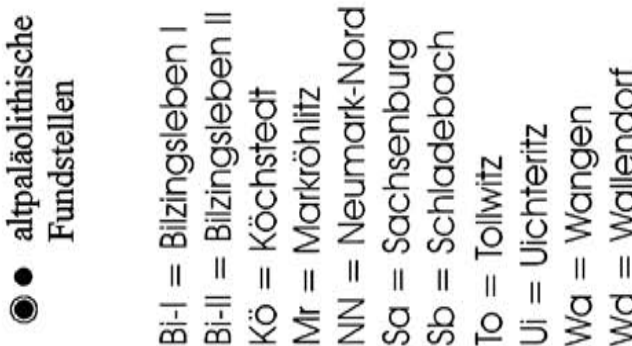
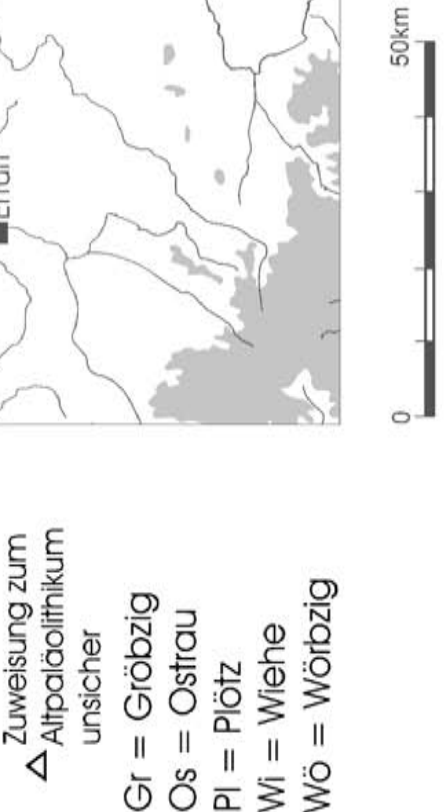
Abschlägen und Kernen fanden sich auch zwei Abschlagsgeräte.

Zwischen 1997 und 2004 wurde die Fundsuche vor allem durch Dr. H. Hartmann, A. Pustlauck, beide Leipzig, sowie W. Bernhardt und A. Rudolph fortgesetzt. Dabei fanden sich an derselben Stelle weitere Artefakte. Alle bisher in den an der Oberfläche ausstreichenden Schottern entdeckten Artefakte stammen aus einem nur etwa $100 \mathrm{~m}$ langen Bereich des nördlichen Talhanges des Röhlitzbaches. Mehrere Begehungen an anderen Stellen, an denen die Schotter oberflächlich verbreitet sind (z.B. am südlichen Abhang des Prießigberges sowie großflächig südwestlich von Uichteritz) blieben erfolglos. Im Fundbereich liegen die Schotter bei ca. 130/135 $\mathrm{m} \mathrm{NN}$ und damit etwa im Niveau der Oberen Schotter in der 1,5 km östlich dieser Fundstelle befindlichen Kiesgrube Uichteritz, die nach Meng \& Wansa (2005) bei 128-133,5 m NN angetroffen werden.

$\mathrm{Da}$ die bis 1988 geborgenen Steinartefakte gegenwärtig größtenteils nicht zugänglich sind, können im Folgenden nur die seit 1997 aufgefundenen Stücke etwas näher charakterisiert werden. Es handelt sich um Artefakte aus einem grauen bis dunkelgrauen nordischen Feuerstein, die überwiegend deutliche Verrundungsmerkmale zeigen. Sie besitzen zumeist eine glänzende, teilweise fleckig weiße oder weißbläuliche Patina. Verschiedene Stücke weisen eine Carbonatkruste auf.

Die Kollektion umfasst bisher 13 Abschläge, 3 Kerne, ein Kernfragment, ein Abschlagfragment mit partieller Retusche (Abb. 4, 6), einen Abschlag mit verdünntem Rücken, aber unbearbeiteter Kante (Abb. 4, 1) und ein triederartiges Stück (Abb. 4, 7). Zusätzlich liegt noch ein Abschlag vor, den J. Thum 1988 auf der Feldoberfläche geborgen hatte (Abb. 4, 2). Die Abschläge und Kerne zeigen ähnliche Dimensionen und Merkmale wie jene von Uichteritz. Zwei Abschläge weisen jedoch eine facettierte Schlagbasis auf (Abb. 4, 1. 2). Einer davon erinnert mit seiner regelmäßig-ovalen Form an einen Zielabschlag (Abb. 4, 2). Besonders das triederartige Artefakt (Abb. 4, 7) verdient weitere Aufmerksamkeit. Es handelt sich zwar nicht um einen Trieder im engeren Sinne, da eine Fläche unbearbeitet ist, doch stellt es ein bifaziales Gerät dar. Diese kommen im mitteldeutschen Raum allgemein selten vor (LAURAT 2003), sind aber beispielsweise auf der bereits erwähnten Fundstelle von Wallendorf ebenfalls mit einigen Stücken vertreten (LAURAT, RUdoLPH \& Bernhardt 2004).

\section{Bedeutung der Funde von Uichteritz und Markröhlitz}

Im mitteldeutschen Raum konnten bisher 11 sicher altpaläolithische Fundstellen entdeckt werden (zusammenfassende Übersichten bei Toeprer 1968, 1970; Mania 1995, 1997) (Abb. 5). Diese gehören alle in den zwischen dem Ende der Elsterkaltzeit und Beginn der Saalevereisung liegenden Zeitraum, erlauben aber zumeist keine sichere feinstratigraphische Zuweisung. Mit den hier vorgestellten Artefaktfunden aus der Kiesgrube Uichteritz liegt nicht nur ein weiterer altpaläolithischer Fundkomplex aus Mitteldeutschland vor. Es sind möglicherweise die ältesten bisher bekannten Funde dieses Raumes. Sie stammen aus Schottern, die wahrscheinlich in der späten Elster-Kaltzeit abgelagert wurden. Ähnliches gilt für das Inventar von Markröhlitz, allerdings ergeben sich bei diesem Einschränkungen dadurch, daß es sich bei den meisten der hier geborgenen Artefakte um Oberflächenfunde handelt. Sie liegen zwar in den am Hang des Röhlitzbaches ausstreichenden Schottern, können aber im Einzelfall natürlich auch jünger sein. STEINMÜLLER (1982) weist auch die Artefaktfunde von Wangen im Unstruttal der späten Elster-Kaltzeit zu. Mit diesen Fundkomplexen besitzen wir die 
frühesten Nachweise für die Anwesenheit von Menschen in Mitteldeutschland. Das macht sie so bedeutsam. Sichere Funde, die in die Zeit vor der elstereiszeitlichen Inlandvereisung zurückreichen, sind hier bisher noch nicht entdeckt worden. Funde aus der folgenden HolsteinWarmzeit sind von Memleben (Weber 1977; Mania 1997) und aus der Bilzingsleben-I-Travertin-Terrasse (MANiA 1997) bekannt, während der bedeutende Homo-erectus-Lagerplatz von Bilzingsleben II (MaNia 1997) und das umfangreiche Inventar von Wallendorf (LAURAT, Rudolph \& Bernhardt 2004) sowie der benachbarte Fundplatz von Tollwitz (LAurat et al. 2004) sogar als noch jünger angesehen werden müssen, aber ebenfalls noch dem Zeitraum zwischen der Holstein-Warmzeit und dem ältesten Eisvorstoß des Saale-Komplexes angehören.

\section{Nachtrag}

Seit der Manuskriptabgabe (Januar 2005) konnte die Fundkollektion aus Uichteritz bei regelmäßigen weiteren Begehungen um folgende Artefakte vermehrt werden: 4 Abschläge, 6 Kerne und zwei Geräte. Letztere können als ein Abschlag mit einer Doppelbucht auf der Ventralseite und einem grob bearbeiteten Rücken sowie als ein pickartiges Kerngerät angesprochen werden. Die Stücke fügen sich gut in das bisher bekannte Artefaktspektrum ein. Das pickartige Stück ähnelt sehr einem Gerät aus dem altpaläolithischen Fundkomplex von Tollwitz (LaUrat et al. 2004, Taf. 12,1), das allerdings größer ist. Von der benachbarten Markröhlitzer Fundstelle liegen ebenfalls mehrere Neufunde vor: ein Abschlag, zwei angeschlagene Feuersteinstücke sowie ein Abschlag mit einer grob retuschierten Schneide und artifiziellem Rücken.

\section{Schriftenverzeichnis}

FritsCh, K. von (1901): Excursion in die Umgebung von Halle. - Zeitschr. d. Dt. Geol. Ges. 53, Verhandl.: 66-86; Berlin.

Laurat, T. (2003): Bifaziale Geräte im Altpaläolithikum Mitteldeutschlands. - In: BuRdukiewicz, J. M., Fiedler, L., Justus, A., HeIn Rich, W.-D. \& BrüHL E. [Hrsg.]: Erkenntnisjäger. Festschrift für Dietrich Mania. - Veröff. d. Landesamtes f. Archäologie Sachsen-Anhalt - Landesmus. f. Vorgesch., 57/II: 357-371; Halle (Saale).

Laurat, T., Rudolph, A. \& Bernhardt, W. (2004): Zu den Gerätetypen der altpaläolithischen Fundstelle Wallendorf (SachsenAnhalt). - Archäol. Korr. Bl., 34: 1-19; Mainz.

Laurat, T., Rudolph, A., Bernhardt, W. \& Schneemilch, M. (2004): Altpaläolithische Feuersteinartefakte von Tollwitz, Ldkr. Merseburg-Querfurt. - Jschr. mitteldt. Vorgeschichte, 88: 1-22; Halle (Saale).

Mania, D. (1973): Paläoökologie, Faunenentwicklung und Stratigraphie des Eiszeitalters im mittleren Elbe-Saalegebiet auf Grund von Molluskengesellschaften. - Beih. z. Zeitschr. Geologie, 78/79: 1-175; Berlin.

- (1995): The earliest occupation of Europe: The Elbe Saale region. - In: Roebroeks, W. \& Van Kolfschoten, Th. [eds.]: The earliest occupation of Europe: Proceedings of the European science Foundation Workshop in Tautavel. - Acta Praehistoria Leidensia: 85-101; Leiden.

- (1997): Altpaläolithikum und frühes Mittelpaläolithikum im Elbe-Saale-Gebiet. - In: FIEDLER, L. [Hrsg.]: Archäologie der ältesten Kultur in Deutschland. - Mat. Vor- u. Frühgesch. Hessen, 18: 86-194; Wiesbaden.

Meng, S. \& Wansa, S. (2005): Lithologie, Paläoökologie und Stratigraphie des Mittelpleistozäns von Uichteritz im Markröhlitzer Tal (Lkr. Weißenfels/Sachsen-Anhalt). 
- Eiszeitalter u. Gegenwart, 55: 174-214; Hannover [dieses Heft].

Schulz, W. (1962): Gliederung des Pleistozäns in der Umgebung von Halle (Saale). - Beih. z. Zeitschr. Geologie, 36: 1-69; Berlin.

- (1963): Abriß der Pleistozänstratigraphie in der Umgebung von Halle (Saale). - Jschr. mitteldt. Vorgeschichte, 47: 37-56; Halle (Saale).

Siegert, L. \& Weissermel, W. (1911): Das Diluvium zwischen Halle a.S. und Weißenfels. - Abh. d. Königl. Preuß. Geol. Landesanstalt, NF 60: 350 S.; Berlin.

Steinmüller, A. (1982): Die stratigraphische Einstufung der pleistozänen Schichtenfolge und des Clactoniens von Wangen im Unstruttal. - Zeitschr. geol. Wiss., 10: 745758; Berlin.

Toepfer, V. (1968): Das Clactonien im SaaleMittelelbgebiet. - Jschr. mitteldt. Vorgeschichte, 52: 1-26; Halle (Saale).

- (1970): Stratigraphie und Ökologie des Paläolithikums. - In: RichteR, H. [Hrsg.]: Periglazial-Löß-Paläolithikum im Jungpleistozän der Deutschen Demokratischen Republik. - Petermanns Geograph. Mitt., Erg., 274: 329-422; Gotha/Leipzig.

Weber, T. (1977): Clactonienfunde von Memleben, Kr. Nebra. Ausgr. u. Funde, 22: 195199.

Weissermel, W. (1908): Erläuterungen zur Geologischen Karte von Preußen und benachbarten Bundesstaaten, Lief. 146, Blatt Weißenfels. - 47 S.; Berlin (Königl. Preuß. Geol. Landesanstalt).

Wüsт, E. (1901): Ein fossilführender Saalekies bei Uichteritz bei Weißenfels. - Zeitschr. f. Naturw., 74: 65-71; Stuttgart.

\section{Anmerkungen}

1) Sie darf nicht mit einer weiteren Kiesgrube bei Uichteritz, nämlich der Ködelschen Grube am nordwestlichen Rand des Dorfes, verwechselt werden, die bis zum Beginn des 20. Jh. in der (saaleeiszeitlichen) Hauptterrasse der Saale bestand und aus der zahlreiche faunistische Funde (warmzeitliche Mollusken; Palaeoloxodon antiquus, Stephanorhinus kirchbergensis, evtl. Mammuthus trogontherii) bekannt sind (v. Fritsch 1901, WÜst 1901, Weissermel 1908, Siegert \& Weissermel 1911). Mania (1973) nimmt aufgrund ihrer etwas höheren Lage an, dass die Kiese, die hier aufgeschlossen gewesen waren, tatsächlich dem (älteren) Corbicula-Horizont entsprechen.

$\left.{ }^{2}\right)$ Die Fundsuche erfolgte vor allem durch W. Bernhardt und A. Rudolph. M. Schneemilch, Merseburg, A. Pustlauck und Dr. H. Hartmann, beide Leipzig, waren jeweils mehrfach an den Begehungen beteiligt, an einzelnen weiteren wirkten außerdem Prof. D. Schäfer, Innsbruck, Dr. T. Weber, Magdeburg, Dr. J. Thum, Dresden, und T. Laurat mit. Geologische Arbeiten und Einschätzungen erfolgten durch Prof. L. Eißmann, Leipzig, Dr. S. Wansa und S. Meng, beide Halle (Saale), R. Wimmer, Roitzsch, sowie M. Krbetschek und A. Dietrich, beide Freiberg. Für ihre Beteiligung an den Geländearbeiten sei an dieser Stelle den genannten Kollegen und Freunden gedankt.

$\left.{ }^{3}\right)$ Für eine Begutachtung der wenigen Größsäugerreste danken wir Herrn Dr. H.-J. Döhle, Landesamt für Denkmalpflege und Archäologie Sachsen-Anhalt, Halle (Saale).

$\left.{ }^{4}\right)$ Die Feuersteinartefakte sind in der Regel deutlich von den in größerer Zahl in den Flußschottern vorkommenden artefaktähnlichen Naturstücken zu unterscheiden, ebenso von Feuersteinen, die beim Kiesabbau beschädigt 
wurden und manchmal an Artefakte erinnern. Lediglich bei einem kernartigen Stück ist der artifizielle Charakter nicht eindeutig. Es wurde deshalb bei der Merkmalanalyse nicht berücksichtigt und nicht abgebildet. Alle hier vorgestellten Funde aus Uichteritz und Markröhlitz befinden sich in der Sammlung des Landesamtes für Denkmalpflege und Archäologie Sachsen-Anhalt, Halle (Saale).
Abbildungsnachweis: Abb. 1-4: W. Bernhardt, Abb. 5: T. Laurat (Kartengrundlage: J. Kanews, Institut für Prähistorische Archäologie der Martin-Luther-Universität Halle-Wittenberg) 